\title{
URUGUAY: ¿DÓNDE ESTÁ El PILOTO? A DOS AÑOS DE GOBIERNO DEL PRESIDENTE MUJICA (2010-2011)
}

\author{
Uruguay: Where is the Pilot? Two years after the \\ inauguration of president Mujica (2010-2011)
}

\author{
JUAN ANDRÉS MORAES \\ Universidad de la República
}

ALEJANDRO GUEDES

Universidad de la República

DIEGO LUJÁN

Universidad de la República

\begin{abstract}
RESUMEN
Este artículo analiza la coyuntura política de Uruguay durante 2010 y 2011. Para ello, el trabajo se divide en cinco secciones. La primera repasa el contexto macroeconómico de mediano plazo, así como algunos indicadores vinculados a la pobreza y la distribución del ingreso. La segunda sección se centra en la asunción del nuevo gobierno electo en las elecciones nacionales de 2009. En particular, esta sección describe y analiza la elaboración de la agenda del nuevo gobierno liderado por el presidente José Mujica, la definición de un nuevo gabinete y la búsqueda de acuerdos interpartidarios para el establecimiento de consensos de larga duración en algunas áreas de política pública definidas por el propio Presidente. Todo ello, en el marco de un análisis sistemático sobre la forma en que el Presidente gestiona la agenda de gobierno dentro y fuera de su partido, así como adentro del gabinete y en interacción con el Poder Legislativo. Precisamente, la tercera sección está dedicada a la actividad del Poder Legislativo y en particular a la forma en que el cartel legislativo del gobierno opera la agenda prioritaria del Ejecutivo. La cuarta sección repasa brevemente la realización de elecciones municipales, luego de constituirse en la única innovación político-institucional desde la reforma constitucional de 1996. La quinta sección repasa los asuntos principales del bienio y concluye.
\end{abstract}

Palabras clave: Uruguay, partidos políticos, Ejecutivo, gabinete, Legislativo, elecciones.

\begin{abstract}
This article analyzes the Uruguayan political juncture during 2010-2011. To that end, the paper is divided in five sections. Section one reviews the medium term economic context as well as some indicators related to poverty and income distribution. Section two focuses on the new elected government in 2009. In particular, this section describes and analyzes the agenda setting process defined by president Mujica, the new cabinet appointments and the first steps to achieve multiparty agreements on a set of policy areas defined by the president's agenda. We review these processes, by exploring the way in which president Mujica leads his own agenda and interacts within and outside his own party and the legislature to endorse his policy proposals. Section three focuses on the Legislative branch and the way in which the Executive is able to cartelize the agenda by sponsoring and endorsing legislation. Section four briefly reviews the subnational elections held in 2010, after performing the only institutional innovation since the constitutional reform in 1996. Section five reviews and concludes with the main issues covered during 2010 and 2011.
\end{abstract}




\section{LA HERENCIA BENDITA}

La caída de Lehman Brothers en octubre de 2008 constituye el puntapié inicial de una crisis financiera desencadenada en los Estados Unidos pero que rápidamente se ha difundido a numerosos países de Europa y el resto del mundo. De hecho, la crisis amenaza con transformarse en una depresión inédita en la historia de la Unión Europea, luego del colapso de Grecia y el deterioro de otras economías mayores del bloque. En este contexto, América Latina se ha presentado relativamente al margen de las señales más visibles de la crisis, mostrando un enlentecimiento en la evolución del producto pero sin shocks externos que afecten seriamente sus mercados financieros y la demanda de bienes y servicios.

Uruguay no es una excepción en América Latina. Durante el periodo 2004-2011, el país ha mostrado señales de crecimiento sostenido con una tasa promedio anual del PIB del $5,9 \%$, lo cual indica que la economía uruguaya se encuentra en su mejor momento de los últimos sesenta años. No obstante, este alto crecimiento fuertemente inducido por factores externos centrados en la demanda de commodities con precios al alza y la demanda interna de bienes y servicios no es nuevo para el país. Uruguay ya ha transitado en el pasado por otros periodos de bonanza económica, solo que existen algunas diferencias importantes de este periodo respecto a otros de crecimiento sostenido.

Al menos cuatro diferencias resultan fundamentales. Por un lado la durabilidad del periodo de crecimiento. Si la programación macroeconómica de mediano plazo es correcta y no se ve limitada por el impacto del deterioro en las economías de la Unión Europea, en 2013 Uruguay culminará una década completa de alto crecimiento económico. Un segundo rasgo destacable estriba en la vulnerabilidad de la economía uruguaya respecto a periodos anteriores. Desde 2005, el país ha hecho un esfuerzo sistemático en la creación de reglas e instituciones dedicadas a limitar el impacto de shocks externos, en el marco de una economía muy abierta a la región y el mundo. Desde la creación de una oficina de gerenciamiento de deuda en el Ministerio de Economía y Finanzas (MEF en adelante) hasta el seguro de depósitos asentado en el Banco Central del Uruguay (BCU), el país muestra un gerenciamiento del programa económico adecuado a las características y en particular a la vulnerabilidad de la economía uruguaya en el concierto internacional. Prueba reciente de ello es el impacto de la crisis financiera norteamericana de 2008. En 2009, Uruguay fue el país de América Latina que verificó la salida más rápida y con mayor nivel de crecimiento, tras la crisis internacional desatada en los Estados Unidos.

En tercer lugar, es importante destacar que a diferencia de lo ocurrido en periodos anteriores de alto crecimiento económico, el país históricamente había mostrado tasas muy bajas de inversión doméstica y extranjera directa. Esta tendencia en la historia económica uruguaya ha sido progresivamente revertida, tanto por un nivel sostenido de inversión nacional, así como un flujo creciente de inversión externa directa. Algunas de estas inversiones pueden ser claramente identificables, como las empresas fabricantes de pasta de celulosa Botnia (UPM) y Montes del Plata. Estos emprendimientos han mostrado un volumen de inversión inédito (como porcentaje del PIB), y se suman al flujo constante de inversionistas de la región en el campo productivo, mayormente dedicados a la producción agroindustrial. 
En cuarto lugar y también a diferencia de lo observado en otros periodos recientes de crecimiento sostenido, Uruguay ha mostrado una caída consistente a través del tiempo en su tasa de desempleo, llegando en 2011 al valor más bajo en la serie consignada en el Cuadro 1. Mientras que en periodos de crecimiento reciente inducidos por el ajuste estructural o las reformas institucionales de segunda generación el país fue capaz de crecer con tasas de desempleo comparativamente altas (11\% en promedio para 1990 2009), la tasa promedio entre 2010 y 2011 se situó en el 6,5 por ciento. Este registro bianual también contrasta con los valores observados durante la recesión iniciada en 1997 y la crisis posterior de 2001 y 2002. Adicionalmente, la caída sistemática en el nivel de desempleo ha estado acompañada por una evolución creciente en el salario real de los trabajadores del sector público y privado cercana al 3,5\% para 2011.

Como puede verse, la evolución del crecimiento indica un periodo de continuidad desde 2003 -con el rebote de la crisis de 2002- y en particular desde 2005, con tasas sostenidas y en buena medida extraordinarias de crecimiento del producto. Asimismo, estas tasas excepcionalmente altas para la evidencia histórica uruguaya han estado acompañadas por un desempeño anómalo en al menos dos variables importantes como el desempleo y la inversión. Estas variables habían mostrado escasa elasticidad respecto a la evolución del producto en décadas anteriores y por primera vez registran cambios de magnitud considerable. Otras variables relevantes de la macroeconomía muestran efectivamente un comportamiento menos sorprendente por su tradicional elasticidad respecto al producto, pero no por ello menos destacable. En particular, cabe destacar la evolución reciente del gasto público y el gasto público social.

El Cuadro 1 muestra que la evolución del gasto público y en particular del gasto público social ha verificado un crecimiento notorio asociado a la evolución del producto. Sin embargo, la elasticidad del gasto público y el gasto público social en particular no ha estado por encima de la evolución del producto. Esto indica que la política económica del gobierno, si bien ha estado claramente orientada a incrementar el gasto a través de nuevas inversiones o incrementos de salarios (públicos), ha buscado mantener un criterio de neutralidad respecto a la evolución del producto. Esta conducta moderada en el manejo de la política fiscal explica en buena medida la evolución general que han tenido las cuentas públicas durante los últimos siete años y en particular los primeros dos años del gobierno del presidente Mujica. Durante 2010-2011, el nivel de déficit fiscal se situó en el 1,2\% del PIB.

Por último, un componente crucial del contexto socioeconómico heredado por el Presidente Mujica en marzo de 2010 se centra en el desempeño de algunos indicadores que han sido tradicionalmente muy caros a la estructura programática del Frente Amplio: Pobreza y equidad. El crecimiento de la economía uruguaya durante el gobierno de Vázquez produjo una caída significativa en los niveles de desempleo, que ayudaron al mejoramiento del estándar y calidad de vida de la población. Sin embargo, el comportamiento de estas dos variables está directamente asociado con la evolución del gasto público y en particular del gasto público social. El buen desempeño de la economía estuvo acompañado por políticas de abatimiento de la pobreza e indigencia, mediante programas específicos de transferencias monetarias o el nuevo régimen de Asignaciones Familiares vigente desde 2008. 


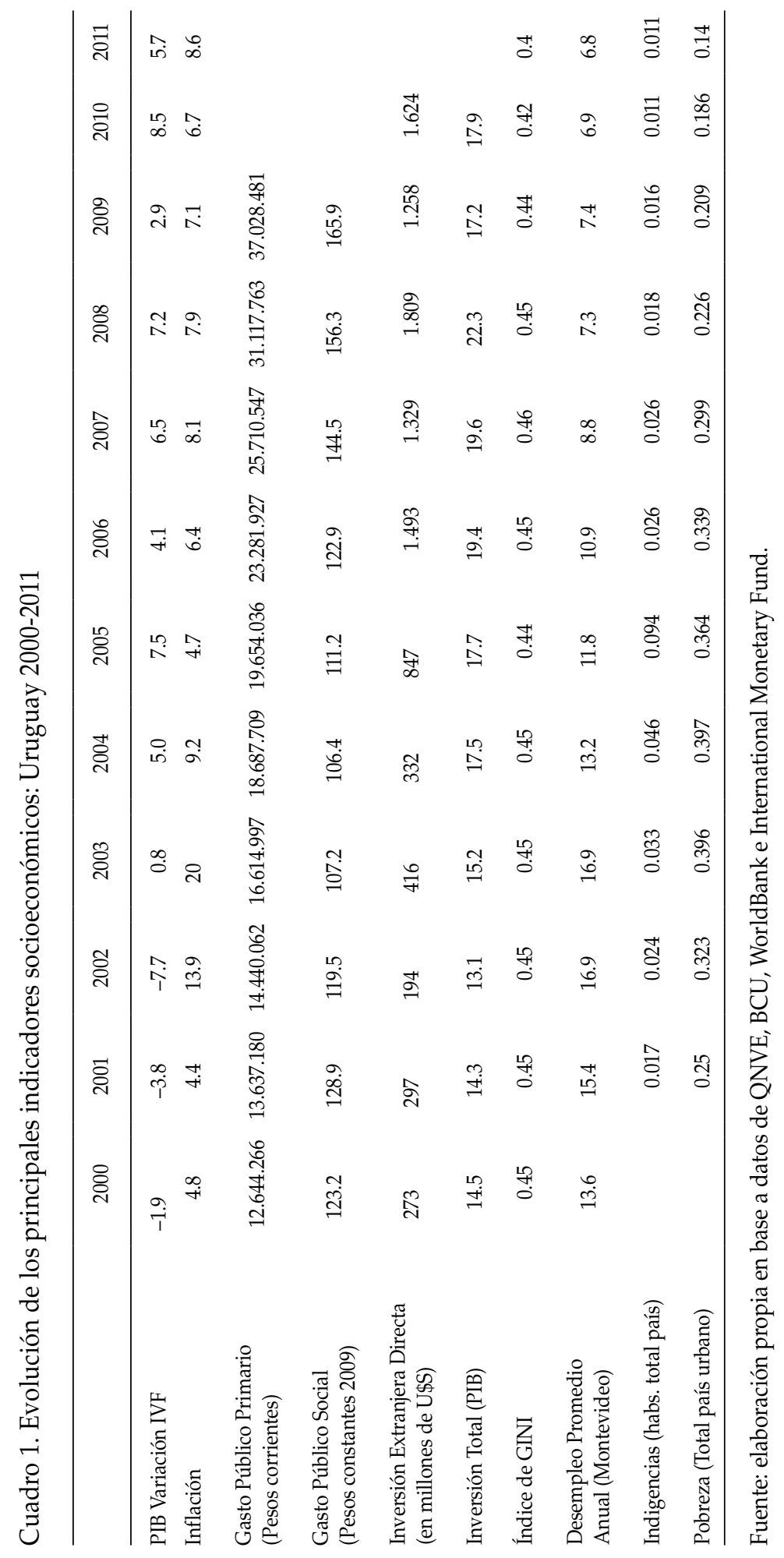


Cuando el presidente Mujica asumió sus funciones en marzo de 2010, el país había logrado reducir la pobreza de 36,4\% a 18,6\% entre 2005 y 2010 (ver Cuadro 1). Durante la actual administración, el gobierno busca abatir por completo la indigencia y seguir con el ritmo de reducción en el número de personas por debajo de la línea de pobreza. Para ello, el actual nivel de empleo y ritmo de crecimiento en el salario real, así como algunas políticas del estado de bienestar uruguayo constituyen los instrumentos fundamentales para la consecución de estos objetivos. Adicionalmente, los niveles de desigualdad, medidos mediante el índice de concentración de ingresos de Gini, muestran que el país no ha logrado revertir seriamente los registros tendientes hacia la concentración verificados durante e inmediatamente después de la crisis de 2001. Sólo a partir de 2007 se verifica una leve tendencia a la baja en la concentración de los ingresos, llegando casi al mismo nivel que en 2003. Nótese que el mejoramiento en la distribución del ingreso no ha verificado mayor impacto a partir de la suba sistemática en el salario real de los trabajadores (con y sin Consejo de Salarios) desde el inicio de la recuperación económica en 2003 (Cuadro 1).

Finalmente, las previsiones de crecimiento de la economía uruguaya para 2012 hacen pensar que si bien el país se verá afectado por la inestabilidad de los mercados internacionales, se prevé que Uruguay crecerá un $(2,2 \%)$ durante el presente año. Si bien la demanda de commodities ha ingresado en una caída importante en los precios internacionales, la demanda interna muestra un gran dinamismo que permite amortiguar los embates de la coyuntura. Además, una sólida situación fiscal, acompañada por el empleo pragmático de instrumentos de política monetaria y cambiaria durante el actual periodo del presidente Mujica, hacen pensar que el país se encuentre razonablemente al cubierto para enfrentar un shock externo marcado por el magro desempeño de las economías de mayor porte a nivel mundial.

\section{EL PODER EJECUTIVO: LA AGENDA DE GOBIERNO}

Luego de ganar las elecciones parlamentarias de octubre y el ballotage de noviembre de 2009, José Mujica fue investido como Presidente de la República por el periodo 2010-2015. En un emotivo discurso de asunción del cargo ante la Asamblea General, el Presidente fue enfático en la selección de prioridades de política pública durante su mandato. Mujica propuso cuatro áreas: educación, energía, medio ambiente y seguridad pública. ${ }^{1}$ Esta definición de prioridades no fue propuesta como una agenda para su partido en el gobierno y mucho menos para su cartel legislativo. Por el contrario, el Presidente propuso que estas cuatro áreas fueran separadas de la agenda convencional del gobierno para ser tratadas aparte, junto a la participación o el concurso de los partidos de oposición.

1 Entre ellas, incluso, estableció una clara jerarquización al decir: "Permítanme un pequeño subrayado: educación, educación, educación. Y otra vez, educación. Los gobernantes deberíamos ser obligados todas las mañanas a llenar planas, como en la escuela, escribiendo 100 veces, "debo ocuparme de la educación". Ver http:/ / www.elpais.com.uy/100302/ pnacio-474229/nacional/lea-el-discurso-de-mujica-en-la-asamblea-general/ 
Las cuatro áreas fueron propuestas como el núcleo de políticas a ser negociadas en el marco de acuerdos interpartidarios que dieran pie a políticas de Estado.

Fuera de este núcleo, otras tres áreas centraron el interés del presidente Mujica en su discurso inaugural. En particular la eliminación de la pobreza extrema o indigencia (con un $2 \%$ de la población en el momento de su asunción), la eliminación de la vivienda precaria mediante la creación de un plan de construcción de viviendas económicas, y la reforma del Estado, a través de un rediseño del aparato estatal orientado a la reducción de sus costos de funcionamiento y una mejora simultánea en la eficiencia. Nótese que estas tres áreas de política no fueron incluidas dentro de las prioridades que el gobierno buscaba consensuar con la oposición. No obstante, fueron objetivos que la oposición aplaudió dentro y fuera de la Asamblea, a través de la opinión de varios líderes partidarios colorados y nacionalistas. La eliminación de la pobreza extrema y la vivienda precaria parecen objetivos cuyo rechazo político se hace difícil en un país poseedor de uno de los mayores estados de bienestar de América Latina.

Virtualmente, la política económica fue puesta a un lado de la agenda porque el gobierno buscó darle continuidad absoluta al curso iniciado durante la administración del ex presidente Vázquez. Recordemos que durante dicho periodo el gobierno optó por la implementación de políticas económicas ortodoxas, cuyo éxito verificado en altas tasas de crecimiento y buen desempeño social hicieron que Mujica se viera limitado a cambiar el curso iniciado durante el gobierno de Vázquez. De hecho, la nominación temprana -luego de la victoria de Mujica en la interna del Frente Amplio- de Danilo Astori (ministro de Economía durante el gobierno de Vázquez) como vicepresidente y la confirmación de Fernando Lorenzo (ex director de la Asesoría Macroeconómica) como futuro ministro de Economía, ambientaron una línea de continuidad evidente en el gobierno de la economía. ${ }^{2}$

En conjunto, las preferencias del nuevo gobierno facilitan los acuerdos interpartidarios, pero también parecen crearle un problema a la oposición. Dado que blancos y colorados han visto con beneplácito la política económica del gobierno del presidente Vázquez y ahora aplauden la continuidad del presidente Mujica, el núcleo de políticas para la búsqueda de acuerdos interpartidarios (en educación, seguridad pública, energía y medio ambiente) deja muy escaso margen para la competencia política entre el gobierno y la oposición.

Desde el ascenso del Frente Amplio al gobierno en 2005, la política económica está fuera del rango de tópicos sobre los cuales debate el sistema político. El diagnóstico de los partidos coincide en que el país goza de un periodo de bonanza inducido por tranquila. Una macroeconomía prolija es un prerrequisito para todo lo demás. Seremos serios en la administración del gasto, serios en el manejo de los déficit, serios en la política monetaria y más que serios, perros, en la vigilancia del sistema financiero. Permítanme decirlo de una manera provocativa: 'vamos a ser ortodoxos en la macroeconomía'". Discurso del Presidente de la República ante la Asamblea General (1/3/2010). Ver http:/ /www.elpais.com.uy/100302/ pnacio-474229/nacional/lea-el-discurso-de-mujica-en-la-asamblea-general/ 
el contexto regional e internacional basado en la demanda externa de commodities, la inversión externa directa y la expansión del mercado interno, lo cual exige un adecuado manejo de instrumentos de política pública como incentivos para la inversión externa, el control de las cuentas fiscales, la apertura comercial con socios de la región y el mundo, un manejo adecuado del endeudamiento mediante la pesificación de la deuda pública y el manejo responsable de la política cambiaria sin afectar la competitividad de la economía uruguaya en su conjunto.

En décadas anteriores, todos estos instrumentos de política económica estaban en disputa y fueron una fuente permanente de debate y competencia política en torno a distintos modelos en pugna entre gobierno y oposición. Sin embargo, el acceso del Frente Amplio al gobierno nacional en 2005 y su línea sostenida de políticas económicas alineada con la de los partidos de la oposición tiene un impacto notorio sobre el rango de políticas públicas sobre las cuales compiten actualmente los partidos del sistema político uruguayo. Prima facie, la determinación de los tópicos de la agenda para el gobierno de Mujica configura un mapa altamente conveniente para el gobierno pero no así para la oposición.

\section{De la agenda a la construcción del gabinete}

En líneas generales, los asuntos de la agenda muestran un compromiso con la estabilidad y la identificación de una serie de políticas donde la alteración del statu quo requiere -a juicio del presidente Mujica- la participación y el consenso de los cuatro partidos con representación parlamentaria (en educación, medio ambiente, seguridad pública y energía). Con esta agenda, el gobierno inició sus funciones con la designación de un nuevo gabinete ministerial. Dicho gabinete presentó al menos tres diferencias notorias en su estructura respecto al primer gabinete del presidente Tabaré Vázquez.

Una primera característica distintiva se asienta en la asignación proporcional de ministros, respecto al peso legislativo de las fracciones que integran el Frente Amplio. El primer gabinete del presidente Vázquez no guardó una relación proporcional entre el tamaño de las bancadas de las fracciones y su peso relativo dentro del gabinete (Chasquetti, 2007). Sin embargo, el presidente Mujica buscó una integración proporcional casi exacta al asignar los puestos de ministro y viceministro en el gabinete (Buquet and Luján, 2011). En este punto, una forma posible de medir y de comparar entre gobiernos es a través del Índice de Congruencia Parlamentaria del Gabinete (ICPG en adelante, Amorim Neto, 1998). El índice varía entre 0 y 1 , siendo [0] "ninguna congruencia entre ambas dimensiones", y [1] "congruencia absoluta entre ambas" (ver Cuadro 3). El índice guarda cierta semejanza con el concepto de proporcionalidad, en la medida que relaciona dos magnitudes y pondera los desvíos entre ambas. Mientras el primer gabinete del presidente Mujica verificó una alta congruencia entre el tamaño legislativo de las fracciones de gobierno y su respectiva participación en el gabinete, con un ICPG de 0,779, el primer gabinete de su antecesor se situó en un ICPG de 0,593.

Una segunda característica del primer gabinete del presidente Mujica es aun más singular, si se tiene en cuenta la integración de otros gabinetes ministeriales, anteriores al gobierno de Tabaré Vázquez. En su primer gabinete, el presidente Mujica diseñó una 
Cuadro 2: Integración del gabinete 2010-2011

\begin{tabular}{|c|c|c|c|c|}
\hline Ministerio & Ministro/a & Sector & Viceministro/a & Sector \\
\hline Interior & Eduardo Bonomi & MPP & Jorge Vázquez & Independiente \\
\hline Relaciones Exteriores & Luis Almagro & MPP & Roberto Conde & PS \\
\hline Economía y Finanzas* & Fernando Lorenzo & FLS & Pedro Buonomo & MPP \\
\hline Defensa** & Luis Rosadilla & CAP-L & Jorge Menéndez & PS \\
\hline $\begin{array}{l}\text { Transporte y Obras } \\
\text { Públicas }\end{array}$ & Enrique Pintado & FLS & Pablo Genta & Independiente \\
\hline Salud Pública*** & Daniel Olesker & PS & Jorge Venegas & PCU \\
\hline $\begin{array}{l}\text { Ganadería, Agricultura } \\
\text { y Pesca }\end{array}$ & Tabaré Aguerre & Independiente & Daniel Garín & MPP \\
\hline $\begin{array}{l}\text { Industria, Energía y } \\
\text { Minería }\end{array}$ & $\begin{array}{l}\text { Roberto } \\
\text { Kreimerman }\end{array}$ & PS & Edgardo Ortuño & VA \\
\hline Educación y Cultura & Ricardo Erlich & MPP & María Simón & Independiente \\
\hline $\begin{array}{l}\text { Trabajo y Seguridad } \\
\text { Social }\end{array}$ & Eduardo Brenta & VA & Nelson Lustaunau & Independiente \\
\hline $\begin{array}{l}\text { Vivienda, Ordenamiento } \\
\text { Territorial y Medio } \\
\text { Ambiente }^{* * * *}\end{array}$ & Graciela Muslera & MPP & Jorge Patrone & FLS \\
\hline Turismo y Deporte & Héctor Lescano & FLS & Lilián Kechichián & FLS \\
\hline Desarrollo Social & Ana Vignoli & PCU & Lauro Meléndez & MPP \\
\hline $\begin{array}{l}\text { Oficina de Planeamiento } \\
\text { y Presupuesto }\end{array}$ & Gabriel Frugoni & Independiente & Jerónimo Roca & Independiente \\
\hline
\end{tabular}

*Pedro Buonomo (MPP) fue sustituido por Luis Porto (FLS).

**Luis Rosadilla (CAP-L) fue sustituido por Eleuterio Fernández Huidobro (CAP-L).

***Daniel Olesker (PS) fue sustituido por su viceministro Jorge Venegas (PCU), cuyo cargo pasó a ocupar Leonel Briozzo ( ).

****Jorge Patrone (FLS) fue sustituido por Raquel Lejtreger ( ).

*****Ana Vignoli (PCU) fue sustituida por Daniel Olesker (PS).

Fuente: www.presidencia.gub.uy

integración de cada cartera donde ministro y viceministro no pertenecieran a la misma fracción del partido de gobierno. Bajo la concepción (implícita) de que el otorgamiento de un ministerio a una fracción conlleva una cooptación de cuadros menores y un potencial manejo inadecuado de los recursos por falta de monitoreo y control político, el presidente Mujica decidió dividir la integración de todas las carteras (menos una: la Oficina de Planeamiento y Presupuesto). De este modo, el Presidente elaboró equipos donde necesariamente el ministro y subsecretario deberían cooperar en la gestión de la cartera. A este punto se agrega una estrategia anexa del Presidente, donde la Oficina de Planeamiento y Presupuesto pasa a constituirse en un equipo económico alternativo, cercano a las preferencias del Presidente. 
Cuadro 3: Índice de Congruencia Parlamentaria del Gabinete

\begin{tabular}{lcccccc}
\hline Fracción & $\begin{array}{c}\text { Leg. Total } \\
\text { (C.Alta+C. }\end{array}$ & $\begin{array}{c}\% \\
\text { Legisladores }\end{array}$ & Ministerios & $\begin{array}{c}\text { \% Gabinete } \\
\text { Fracción }\end{array}$ & Diferencia & ICPG \\
\hline MPP & 30 & 46,2 & 4 & 28,57 & $17,6 \%$ & 0.781 \\
FLS & 15 & 23,1 & 3 & 21,43 & $1,6 \%$ & \\
PS & 11 & 16,9 & 2 & 14,29 & $2,6 \%$ & \\
CAP-L & 3 & 4,6 & 1 & 7,14 & $-2,5 \%$ & \\
PCU & 2 & 3,1 & 1 & 7,14 & $-4,1 \%$ & \\
VA & 2 & 3,1 & 1 & 7,14 & $-4,1 \%$ & \\
& 65 & 100 & 14 & 100,00 & 0,437 & \\
\hline
\end{tabular}

Fuente: Buquet y Luján, 2011.

En tercer lugar, una diferencia notoria respecto al gabinete del primer gobierno del Frente Amplio estuvo dada por el personal reclutado para liderar la función de ministro y viceministro. Mientras que Tabaré Vázquez incorporó a los líderes de las fracciones del Frente Amplio en el gabinete (ver Buquet, 2009; Chasquetti, 2008; Garcé, 2010), la selección del presidente Mujica apeló a figuras de segundo plano en el liderazgo de las fracciones del Frente Amplio. El criterio empleado por Vázquez buscaba mantener una sintonía fina entre el Presidente y su partido, a los efectos de sobrellevar aceitadamente la agenda de gobierno en el Parlamento. Al ser parte del gabinete, los líderes de las fracciones debían negociar directamente con el Presidente, sin tener que enfrentar contingencias en el partido de gobierno o el propio Parlamento. Mujica, en cambio, lleva adelante la doble labor de negociar dentro del gabinete y con los líderes de las fracciones, cuando no con algunos miembros de la bancada del Frente Amplio en el Legislativo.

Es importante destacar que una innovación importante del presidente Mujica respecto a Vázquez se centra en el retorno de la integración multipartidaria (o de la oposición) en los directorios de los entes autónomos y las empresas públicas. Durante el primer gobierno del Frente Amplio, Tabaré Vázquez y los líderes de los partidos de oposición no lograron ponerse de acuerdo en torno a la integración de dichas instituciones. El presidente Mujica consideró que el nuevo ingreso de la oposición en los directorios de varias instituciones públicas redundaría en un mejor relacionamiento con la oposición y un mayor nivel de control sobre la gestión del partido de gobierno.

Luego de dos años de gobierno del presidente Mujica, los resultados de estas tres estrategias en la confección del gabinete no parecen ser del todo positivos. En primer lugar, la proporcionalidad de los integrantes del gabinete respecto al tamaño legislativo de las fracciones parece ser inocua respecto al funcionamiento efectivo del gabinete. El actual gabinete es más proporcional que el del presidente Vázquez y sin embargo han existido numerosas (y notorias) instancias de disputa y bloqueo de políticas públicas de envergadura dentro del propio consejo de ministros. En principio, la proporcionalidad 
no garantiza un mejor relacionamiento entre los miembros del gabinete y mucho menos de las fracciones que componen al partido de gobierno. ${ }^{3}$

En segundo lugar, la integración mixta de las carteras (o lo que algunos analistas han dado en llamar integración horizontal, en oposición a la integración vertical de una cartera en manos de un solo partido o fracción) también ha mostrado un magro resultado respecto a las expectativas del Presidente durante los primeros dos años de gobierno. De hecho, entre finales de 2011 y marzo de 2012 varias carteras han mostrado profundas diferencias a la interna, donde el Presidente ha debido efectuar cambios importantes a nivel de las subsecretarías. El caso más visible ha sido la Subsecretaría del Ministerio de Economía y Finanzas, donde el subsecretario Buonomo mostró distancia respecto a las preferencias de política del ministro Fernando Lorenzo. Alineado al núcleo más cercano de asesores del presidente Mujica, Buonomo pasó a integrar las filas de la Presidencia de la República. Otros casos evidentes de disputa dentro de la cartera se verificaron en los Ministerios de Vivienda, Educación y Ganadería, Agricultura y Pesca. ${ }^{4}$

Por último, no tener al liderazgo de las fracciones dentro del gabinete (como efectivamente ocurrió durante el gobierno de Tabaré Vázquez) muestra que el Presidente debe efectuar un número mayor de instancias de negociación, a los efectos de promover su agenda dentro del gabinete. El liderazgo de las fracciones se encuentra en el Parlamento, pero los ministros, aun siendo personal político de segunda línea, tienen que liderar sus propias áreas de política pública y deben negociar en forma independiente con el Presidente y otros ministros del gabinete. Por este motivo, el Presidente enfrenta una situación de mayor dispersión del poder dentro de su propio gobierno -con un mayor número de agentes de veto-, a pesar de gozar de un apoyo legislativo mayoritario y ser líder de la fracción mayoritaria en el gobierno.

\section{El Gobierno y la gestión de la agenda}

Coherente con las prioridades temáticas establecidas por el presidente Mujica en la inauguración del segundo gobierno del Frente Amplio, la educación pública acaparó rápidamente la atención del gobierno luego del 1 de marzo de 2010. De acuerdo a las prioridades establecidas por el Presidente en su discurso inaugural, la educación debía ocupar un sitial aparte. De hecho, fue concebida como el área más importante de las cuatro en que el Presidente abogó por el establecimiento de acuerdos interpartidarios que dieran lugar a políticas de Estado (o de larga duración). Es así que en mayo de 2010, los partidos ya habían logrado establecer un primer acuerdo básico en materia de educación pública, llamado anodinamente "Documento de Acuerdo sobre Educación" (Bentancur and Mancebo, 2011). Este acuerdo tenía objetivos en varios niveles, así como algunas metas claramente establecidas en torno a la educación inicial (o preescolar) y la educación primaria. Sobre la base de un diagnóstico que indicaba un fuerte rezago

4 La destitución de Garín y el ingreso de Enzo Benech en el MGAP, la destitución de María Simón en el MEC y su sustitución por Óscar Gómez, y la renuncia de Jorge Patrone en el MVOTMA y su sustitución por Raquel Lejtreger. 
en la cobertura de educación preescolar y tasas llamativamente altas de deserción y repetición escolar, el documento de acuerdo interpartidario atacó estos dos objetivos con metas claramente establecidas.

El acuerdo de 2010 significó un logro importante para los objetivos del gobierno. Sin embargo, 2011 se iniciaría con una inesperada turbulencia en materia de políticas educativas. A fines de 2010 el gobierno divulgó los resultados de las pruebas PISA (Programme for International Student Assessment) de evaluación estandarizada de conocimiento. Los resultados no fueron los esperados, dado que, si bien posicionaban positivamente a Uruguay en el concierto latinoamericano, calificaban muy por debajo de las expectativas a nivel mundial. Además, estos resultados contrastaban fuertemente con el volumen de recursos vertidos en el sector educativo durante el quinquenio anterior, mostrando un resultado ineficiente en función de la evolución del gasto. La divulgación de los datos desató un intenso debate dentro y fuera del sistema político, pero en particular entre el gobierno y la oposición. Este debate, canalizado fuertemente a través de los medios pero también por la vía parlamentaria mediante una interpelación al ministro de Educación y Cultura (el 14/9/2011), duró casi todo el año, revelando que la oposición, lejos de acercarse al gobierno, decidió marcar una fuerte oposición a la gestión gubernamental en materia educativa. Durante este período, el gobierno se abocó a la implementación de la Ley General de Educación aprobada por el primer gobierno frenteamplista en 2008. No fue hasta finales de 2011 y los primeros meses de 2012 en que el propio Presidente buscó un nuevo acuerdo con la oposición en materia educativa.

Otra de las áreas de política que el presidente Mujica propuso separar de la agenda para ser tratada como política de Estado es el sector energético. En este punto, el gobierno inició sus actividades sin mayores propuestas, hasta que el director de la Dirección Nacional de Energía (Ramón Méndez) y en menor medida el Presidente de la República comenzaron a insinuar un debate en torno al sector. En líneas generales, el gobierno proponía una diversificación de la matriz energética a los efectos de disminuir su dependencia de la importación de petróleo y gas. En este contexto, el gobierno declaró estar dispuesto a rever la legislación actual que prohíbe la instalación de plantas atómicas en territorio uruguayo. Sin embargo, el tsunami y posterior catástrofe atómica en Japón en marzo de 2011 echaron por tierra cualquier posibilidad de poner sobre la mesa el debate sobre la energía atómica. Luego de esta instancia fallida, la agenda en materia energética y el manejo de recursos naturales encallarían en otro sector con notorio impacto ambiental: la minería. A mediados de 2011, la empresa de capital indio Aratirí recaló en Uruguay con el objetivo de explotar la producción de hierro, mediante la instalación de una mina a cielo abierto en el pueblo Valentines.

En este caso, el Presidente quedó virtualmente solo en la promoción de la minería en las condiciones que proponía la empresa india. Un permanente desgaste en los medios, con información indeterminada sobre las características de la inversión y su impacto ambiental, le hizo ganar no sólo la oposición de Blancos y Colorados sino también la del propio Frente Amplio. De hecho, las vacilaciones del Presidente lo llevaron a proponer un plebiscito sobre el asunto de la minera Aratirí, provocando las molestias de todo el sistema político. En ese contexto, el Presidente designó al ex subsecretario de Economía (Pedro Buonomo) para liderar las negociaciones entre el gobierno y la oposición, a los efectos de 
lograr un acuerdo interpartidario y eventualmente una ley sobre la producción minera de gran porte en Uruguay. A pesar de que el presidente Mujica pretendió inicialmente tratar las políticas energéticas como políticas de Estado, no supo liderar el proceso y de hecho la legislación que actualmente se aprestan a aprobar todos los partidos en el Parlamento no tuvo origen en la Presidencia de la República.

Por último, la seguridad pública también fue seleccionada por el presidente Mujica como una de las cuatro áreas de política prioritarias para su gobierno (Vila, 2011). Al igual que en educación y energía, el gobierno buscó en 2010 establecer acuerdos interpartidarios con la oposición y formó una comisión con dicho propósito. La comisión llegó a ciertos acuerdos importantes que se vieron plasmados en políticas y recursos posteriormente incluidos en la ley de presupuesto para el periodo 2010-2014 (18.729). Sin embargo, la cooperación tuvo un límite muy cercano en el tiempo, dado que hacia fines de 2010 el líder del partido y senador colorado Pedro Bordaberry decidió buscar un camino de competencia en el área de la seguridad pública. El gobierno no mostraba resultados satisfactorios en algunos indicadores importantes para las estadísticas recogidas en el Observatorio de la Seguridad Pública y los medios de comunicación ayudaban a difundir amplia e insistentemente actos delictivos de diverso tipo (algunos de los cuales involucraban a menores infractores). Precisamente, el senador Bordaberry decidió proponer una reforma constitucional orientada a la baja de la imputabilidad de los menores, actualmente situada en los 18 años. ${ }^{5}$ La campaña busca recoger firmas a los efectos de plebiscitar junto con las elecciones de 2014 una reforma de la legislación actual que inhibe la imputabilidad de menores.

Desde la adopción de este camino por parte del senador Bordaberry, el Partido Colorado decidió competir sobre este eje de política pública, en vez de cooperar mediante la búsqueda de nuevos acuerdos (más allá de los que marcaron el inicio de la gestión del presidente Mujica). Este paso importante dejó en una posición incómoda al Partido Nacional, el cual se dividió en su apoyo a la estrategia del senador Bordaberry. El herrerismo, por un lado, optó por apoyar el camino del Partido Colorado, mientras que Alianza Nacional (liderada por el senador Larrañaga y tradicionalmente más cercano al presidente Mujica) optó por no acompañar la estrategia del Partido Colorado. ${ }^{6}$

\section{El liderazgo presidencial de la agenda y el gobierno}

Uno de los asuntos que ha concitado mayor interés de analistas y políticos durante los últimos dos años ha sido el peculiar estilo de liderazgo político ejercido por el presidente Mujica. Su estilo directo y fuera de protocolo ha sido siempre uno de los factores más

5 El Partido Colorado había firmado el acuerdo interpartidario sobre seguridad ciudadana, pero solicitó expresamente que constara en el acuerdo que su partido no había logrado el apoyo del gobierno en el mantenimiento de los antecedentes de los menores infractores y la baja en la edad de imputabilidad en el caso de delitos gravísimos.

6 En el área de Medio Ambiente, el gobierno también logró firmar un acuerdo con la oposición, luego de que una comisión multipartidaria se pusiera de acuerdo sobre cuatro ejes: Gestión ambiental, cambio climático, participación pública e institucionalidad ambiental. El 7 de junio de 2010 los partidos firmaron el acuerdo en el Parlamento. 
importantes detrás de su reputación y popularidad. Ya desde su pasaje como legislador se había caracterizado por mantener un diálogo fluido y en los mismos términos (de igual a igual) con empresarios, productores y trabajadores. Lo mismo ocurrió durante su paso por el Ministerio de Ganadería, Agricultura y Pesca. Fue, de acuerdo a todos los sondeos de opinión pública, el más popular de todos los ministros del gabinete. Indudablemente, este peculiar estilo de ejercer la actividad política ha sido su sello personal y por extensión la clave de su éxito electoral.

Sin embargo, en el marco de sus funciones como Presidente de la República, el estilo característico de Mujica le ha reputado (tanto a él como Presidente y al Frente Amplio como gobierno) más problemas que beneficios, en una opinión pública que en buena medida apreciaba positivamente su estilo. Más adelante observaremos en profundidad la evolución de la opinión pública durante los primeros dos años del gobierno de Mujica. Por ahora, es importante hacer notar que la forma en que el Presidente ha liderado su propia agenda de gobierno le ha reputado costos importantes, no sólo a nivel de apoyo en la opinión pública, sino también en el procesamiento de algunas políticas prioritarias para el gobierno. En particular, la forma de liderar la agenda de gobierno ha mostrado a un presidente que generalmente explicita sin mayor detalle algunos asuntos que su círculo más cercano de asesores, el gabinete o algún ministerio en particular, viene diseñando y eventualmente negociando en la interna del gobierno.

En teoría, el acto presidencial de acudir al público (o going public en la frase acuñada por Samuel Kernell, 1993) es consecuencia directa de un déficit de apoyo político. Un presidente políticamente débil o con escaso apoyo parlamentario, ya sea de su partido o de una coalición de partidos, tiene incentivos para acudir al público en general, a los efectos de imputar o asignar una responsabilidad a quienes no lo apoyan. Esto es particularmente cierto cuando el presidente tiene un capital político (de apoyo popular) que le permite tener suficiente autoridad frente a sus detractores. Sin embargo, un presidente políticamente fuerte o continentado por un cartel legislativo cuya mayoría ha demostrado ser particularmente disciplinada, no necesitaría acudir al público en apoyo de sus preferencias de política. Al momento de iniciar su mandato, el presidente Mujica no sólo contaba con un capital político en la opinión pública en general, sino que además fue electo junto a una mayoría parlamentaria que lo dejaba fuera de contingencias en el Legislativo. ¿Por qué entonces el presidente Mujica ha empleado sistemáticamente esta estrategia de "ir al público"?

Este camino ha tenido como resultado sistemático la aparición de agentes de veto durante el proceso de negociación de políticas públicas de gran relevancia para el Frente Amplio, pero también para la oposición, con quien el propio Presidente busca acuerdos interpartidarios para la concreción de políticas de Estado. Las acciones del Presidente en momentos frecuentemente inoportunos de la elaboración y negociación de políticas, han levantado en varias instancias una oposición o reacción anticipada tanto en el seno del partido de gobierno como entre la oposición y las organizaciones sindicales y empresariales. Nuevamente: ¿Por qué el Presidente emplea sistemáticamente el camino de "ir al público" (cuando no lo necesita)? 
Vale la pena destacar algunas instancias en las que el estilo del Presidente ha sido contraproducente para sus propios objetivos. A poco tiempo de asumir sus funciones como Presidente de la República, Mujica propuso sin mayor detalle la creación del Ministerio de Gobierno. Este nuevo ministerio estaría a cargo de la coordinación interministerial y en los hechos buscaba convertir al nuevo ministro en una suerte de primer ministro -o primus inter pares- dentro del gabinete. La idea fue inmediatamente bombardeada desde filas del Frente Amplio y la oposición, y el Presidente no insistió con su idea. Simplemente, generó un ambiente adverso a sus preferencias sin tener un proyecto concreto. De haberlo tenido, como en otras oportunidades en que adelanta tópicos que su gabinete está negociando arduamente, hubiera persistido en la idea de crear el Ministerio de Gobierno. Sin embargo, la idea se desvaneció rápidamente.

Poco después, el presidente Mujica comenzó a reunirse con el Dr. Daniel Ferrere, director de un afamado bufete de abogados de Montevideo, con quien buscaba asesorarse sobre los posibles contenidos de una reforma del Estado. El fruto de sus interacciones fue rápidamente revelado a los medios y su propia audición semanal de radio, donde explicitaba algunas ideas para llevar a cabo durante su mandato. Este paso era aun más riesgoso que el anterior, dado que el Presidente apelaba al asesoramiento de un abogado sin experiencia alguna en materia de reformas al sector público, pero que además tenía preferencias claramente antiestatistas en un país donde aproximadamente el $80 \%$ de la población está a favor del Estado como propietario de servicios públicos y además se halla satisfecha con la provisión de sus servicios. Las ideas (no proyectos) de eliminación de la inamovilidad de los funcionarios públicos, los salarios por productividad y otras ideas rupturistas tuvieron un rápido final, luego de la muerte trágica del Dr. Ferrere en un accidente aéreo y la crítica efectuada por los sindicatos del sector público y parte de la interna del Frente Amplio.

Un tercer episodio durante 2011 ocupó la atención de los medios con una nueva irrupción del Presidente en la opinión pública. En este caso, Mujica decidió cargar las baterías contra una institución de la economía uruguaya: el secreto bancario. Esta legislación llegó de la mano de la dictadura en 1974, y fue durante largo tiempo uno de los objetivos a eliminar por parte de la estructura programática del Frente Amplio. Dado que el secreto bancario promueve el flujo internacional del capital bancario y no productivo, esta regulación fue históricamente criticada por la coalición de izquierda. Sin embargo y por la vía de los hechos, el primer gobierno del Frente Amplio logró convivir sin problemas con el secreto bancario y de hecho le mostró a la propia izquierda que un gobierno de este signo ideológico no sólo puede captar los mayores niveles de inversión externa directa en la historia del país, sino que también puede convivir con una reforma impositiva que grava los ingresos personales mediante el impuesto a la renta. ¿Por qué cargar ahora las baterías contra el secreto bancario? La idea fue rápidamente desechada, tras una furibunda crítica de la oposición, a la que se sumó el propio equipo económico con el vicepresidente a la cabeza. ${ }^{7}$

7 Dada la gran afinidad entre Cristina Fernández de Kirchner y el presidente Mujica, la oposición política uruguaya rápidamente levantó la suspicacia de que la voluntad del Presidente uruguayo estaba alineada a 
El punto más crítico en los problemas del liderazgo presidencial de Mujica se produjo en agosto de 2011 con el manejo de la cuestión de los derechos humanos. Este ha sido un tema recurrente en la agenda del sistema político desde la restauración democrática en 1985. El origen de la competencia política en este tema deriva de la Ley de Caducidad de la Pretensión Punitiva del Estado (15.848), comúnmente conocida como ley de caducidad. Básicamente, la ley otorga inmunidad a militares y civiles involucrados en la violación de derechos humanos durante la dictadura militar. En dos oportunidades (1989 y 2009), los propulsores de su anulación por la vía plebiscitaria habían perdido en las urnas. Al mismo tiempo, si bien el congreso del Frente Amplio en 2004 aceptó respetar el fallo del plebiscito de 1989 y los preceptos de la ley aprobada durante la transición, se vio enfrentado a una importante presión de la central sindical (PIT-CNT), la cual impulsaba una nueva instancia de anulación durante las elecciones de 2009.

Luego de una nueva derrota electoral en 2009 pero acicateados por el fallo de la Suprema Corte de Justicia -a instancias de la Corte Interamericana de Derechos Humanos-, la bancada legislativa del Frente Amplio decidió promover un proyecto de ley por el cual se dejaba sin efecto la Ley de Caducidad. El Presidente mostró su apoyo y sostuvo que no vetaría la iniciativa legislativa. Sin embargo, existe una sospecha generalizada de que el Presidente forzó a uno de sus legisladores más cercanos a constituirse en veto legislativo para que el proyecto muriera en la Cámara de Diputados. Resulta evidente que el Presidente no quería pagar el costo de oponerse abiertamente al proyecto del Legislativo que anulaba la Ley de Caducidad. Sin embargo, también resulta evidente que el procedimiento deja al Presidente como responsable del veto legislativo impuesto por el diputado Semproni.

Apenas una semana después del estrepitoso fracaso del proyecto de ley interpretativo de la Ley de Caducidad, el presidente Mujica irrumpió en la escena pública con un nuevo asunto que generaría disrupción dentro y fuera de su partido: el impuesto a la concentración de la tierra. La concentración (y en parte la extranjerización) de la tierra ha sido uno de los temas recurrentes en las preocupaciones del Presidente y más en general del MLN (Tupamaros). De hecho, durante su paso como ministro de Ganadería, Agricultura y Pesca (entre 2005 y 2009), Mujica había insistido con esta preocupación en varias oportunidades. Sin embargo, debió ser electo Presidente para promover su proyecto, centrado en un impuesto que grava la concentración en la tenencia de la tierra, cuya recaudación estaría dedicada a cubrir los costos del mantenimiento de la caminería rural (aproximadamente unos sesenta millones de dólares al año).

En esta oportunidad, el Presidente declaró su intención de gravar al sector agropecuario en el marco de una conversación sobre otros temas con periodistas apostados en la puerta del edificio de la presidencia. La noticia tomó por sorpresa no sólo al ministro de Ganadería, Agricultura y Pesca, sino principalmente al ministro de Economía. El impuesto tenía origen en la Oficina de Planeamiento y Presupuesto y no había sido 
negociado. Sin embargo, el propio Presidente declaró enfáticamente "el Presidente no se baja" en tono amenazante ante cualquier posibilidad de veto (dentro y fuera de su partido). Para los sectores del agro y otros operadores del sector privado, el impuesto fue visto como un cambio en las reglas de juego, dado que el equipo económico había dado señales de que la carga impositiva al sector no sería modificada. Para el equipo económico (asentado en el Ministerio de Economía), el impuesto no sólo cambiaba las reglas de juego sino que también violaba la autoridad del Ministerio en el gobierno de la política impositiva. El proyecto fue largamente negociado entre el Ministerio de Economía y la OPP, hasta que un acuerdo político entre el vicepresidente y el propio Presidente abrió el camino para su aprobación en el Parlamento (28/12/2011).

\section{La opinión pública: ¿Un corolario?}

El presidente Mujica logró amalgamar dos hechos independientes pero cruciales para $\mathrm{su}$ victoria en las elecciones de octubre y posteriormente noviembre de 2009. Por un lado, logró de buena manera hacerse heredero de los éxitos de algunas políticas implementadas durante el gobierno del presidente Vázquez, como la política económica y social. Por otro, logró hacer de su excelente imagen pública un candidato presidencial viable para la mayoría del Frente Amplio y electorado uruguayo en su conjunto. Estos dos insumos constituyeron un capital político extremadamente valioso en la asunción del Presidente Mujica, en la medida que le otorgaban legitimidad para la consecución de su agenda de gobierno.

Sin embargo, el devenir de algunos acontecimientos ya mencionados ha provocado un sistemático desgaste de la figura del Presidente y su gobierno en la opinión pública. A diferencia de lo ocurrido con el presidente Vázquez, Mujica ha visto desgastada su figura durante la mitad de su mandato. Como ya hemos visto, el origen de esta erosión podría hallarse en la forma en que se ha visto involucrado en el proceso de diseño y negociación de políticas públicas de gran envergadura. En varias ocasiones, el tipo de liderazgo presidencial llevado a cabo por el presidente Mujica no ha redundado en un beneficio para su propia agenda de reformas, sino que más bien parece haberla inhibido.

Los estudios de opinión pública muestran un claro deterioro de la imagen del Presidente durante 2010-2011. En este período, la opinión pública parece haber iniciado el mandato del presidente Mujica con muy altas expectativas que luego se han ido desvaneciendo. Por ejemplo, en una encuesta publicada por Equipos Mori en febrero de 2010, el 60\% de la opinión pública afirmaba que el gobierno del presidente Mujica sería bueno o muy bueno y apenas un $10 \%$ afirmaba que sería malo o muy malo. En noviembre de ese mismo año, solo un 36\% de la opinión pública declaraba que la gestión se desarrollaba "tal como lo esperaba o por encima de lo esperado" y un 62\% declaraba que la gestión estaba "por debajo o muy lejos de lo esperado" (ver Selios, 2011). Debido a la ausencia de microdatos, es imposible estimar el efecto directo que tiene el tipo de liderazgo ejercido por el presidente Mujica sobre su evaluación. Un presidente puede ser mal evaluado por sus dificultades para hallar soluciones a un problema de política pública o una crisis económica en general. Este parece ser el caso con el manejo que ha realizado el gobierno 
Cuadro 4: Popularidad y simpatía del Presidente Mujica 2010. 2011 (en porcentajes)

\begin{tabular}{lccc}
\hline Popularidad de Mujica & Apr-10 & Nov. 2010 & Jun-11 \\
\hline Simpatía & 66 & 67 & 48 \\
Antipatía & 18 & 17 & 27 \\
No opina/NC & 16 & 16 & 25 \\
Saldo (simpatía/antipatía) & 48 & 50 & 21 \\
\hline Aprobación de la gestión del Presidente Mujica & Apr-10 & Nov. 2010 & Jun-11 \\
\hline Aprueba & 66 & 58 & 39 \\
Desaprueba & 6 & 18 & 39 \\
No opina/NC & 28 & 24 & 22 \\
Saldos & 60 & 40 & 0 \\
\hline
\end{tabular}

Fuente: Elaborado en base a datos de Cifra 2011.

en materia de seguridad pública y educación. Independientemente de los indicadores de desempeño en estas dos áreas, la opinión pública ha sido particularmente crítica con las respuestas o alternativas de política ofrecidas por el gobierno durante los dos primeros años de gobierno del presidente Mujica (ver Selios, 2011; Vila, 2011).

\section{EL PODER LEGISLATIVO}

Las elecciones nacionales de 2009 arrojaron un resultado que le permitió al Frente Amplio acceder a un segundo mandato consecutivo en el gobierno. Sin embargo, el resultado de la elección parlamentaria de octubre le dejaría una importante restricción al partido de gobierno: una coalición mínima ganadora (Chasquetti y Guedes, 2011). Esta configuración mayoritaria hace que cada legislador se constituya en un potencial agente de veto dentro del Frente Amplio. De hecho y como acabamos de señalar con el singular caso que involucró al diputado Semproni en el caso del proyecto interpretativo de la ley de caducidad, existe al menos un ejemplo en que un solo legislador (en una de las Cámaras) puede bloquear la legislación en juego. Desde 2009, el gobierno y en particular el Presidente debe ser capaz de mantener disciplinada a su bancada de legisladores si quiere aprobar su agenda de reformas en el Parlamento. Mujica no necesita los votos de la oposición para lograr las mayorías que le permitan gobernar (ver Cuadro 5). ${ }^{8}$

En este contexto, la oposición debe desarrollar su actividad de contralor a un costo mayor al verificado en gobiernos anteriores. Por un lado, la suma de los tres partidos de oposición no les permite bloquear en el Parlamento la legislación proveniente del Poder Ejecutivo. Ello limita sus posibilidades de colegislar, salvo que sean capaces de

8 Esta configuración permanece intacta, a pesar de la salida (el 27/6/2011) del Frente Amplio del senador Jorge Saravia, perteneciente al MPP. El gobierno mantuvo su cartel legislativo en el Senado, gracias al vicepresidente de la República que oficia de presidente del Senado y la Asamblea General. 
esponsorear legislación junto al cartel mayoritario del gobierno (Ver Magar and Moraes, 2012; Chasquetti and Guedes, 2011). Por otro lado, la fragmentación en el sistema de partidos también afecta su capacidad de coordinación a los efectos de monitorear al gobierno en el Parlamento. Todo ello, agravado por el hecho de que la paridad entre las fuerzas de oposición aumentó tras la elección de 2010, donde el Partido Colorado logró aumentar su caudal electoral. En el marco de esta configuración han discurrido los primeros dos años de gobierno del presidente Mujica, donde el Legislativo ha sido una importante caja de resonancia de la actividad del gobierno y su interacción con la oposición.

Cuadro 5: Composición 47ª Legislatura. 2010-2015

\begin{tabular}{|c|c|c|c|c|c|}
\hline \multirow{2}{*}{ Partido } & \multirow[b]{2}{*}{ Fracción } & \multicolumn{2}{|c|}{$\begin{array}{c}\text { Cámara } \\
\text { de Diputados }\end{array}$} & \multicolumn{2}{|c|}{$\begin{array}{l}\text { Cámara de } \\
\text { Senadores }\end{array}$} \\
\hline & & Escaños & $\%$ & Escaños & $\%$ \\
\hline \multirow{9}{*}{ Frente Amplio } & Espacio 609 & 24 & 48 & 6 & 35 \\
\hline & Asamblea Uruguay & 6 & 12 & 4 & 24 \\
\hline & Partido Socialista & 9 & 18 & 2 & 12 \\
\hline & Democracia Avanzada & 2 & 4 & 1 & 6 \\
\hline & Alianza Progresista & 2 & 4 & 1 & 6 \\
\hline & Nuevo Espacio & 3 & 6 & 1 & 6 \\
\hline & CAP-L & 2 & 4 & 1 & 6 \\
\hline & Vertiente Artiguista & 1 & 2 & 1 & 6 \\
\hline & Cabildo & 1 & 2 & - & - \\
\hline \multirow{4}{*}{ Partido Nacional } & Total & $50(50 \%)$ & 100 & $(55 \%)$ & 100 \\
\hline & U.N.A. & 18 & 60 & 5 & 56 \\
\hline & Alianza Nacional & 12 & 40 & 4 & 44 \\
\hline & Total & $30(30 \%)$ & 100 & $9(29 \%)$ & 100 \\
\hline \multirow{5}{*}{ Partido Colorado } & Vamos Uruguay & 14 & 82 & 3 & 60 \\
\hline & PROBA & 3 & 18 & 2 & 40 \\
\hline & Total & 17 & 100 & 5 & 100 \\
\hline & & $(17 \%)$ & & $(16 \%)$ & \\
\hline & & 2 & & & \\
\hline \multirow{2}{*}{ Partido Independiente } & & $(2 \%)$ & & - & - \\
\hline & & 99 & & 31 & \\
\hline Total & & $(100 \%)$ & & $(100 \%)$ & \\
\hline
\end{tabular}

Fuente: Corte Electoral-Banco de Datos (PRI-FCS). 
El Cuadro 6 muestra la sorprendente regularidad del funcionamiento de las instituciones uruguayas. Obsérvese que las diferencias registradas para los dos primeros bienios de Tabaré Vázquez y José Mujica en el Poder Ejecutivo son casi imperceptibles. De hecho, el Ejecutivo envió casi el mismo número de proyectos y aprobó el mismo número de leyes durante los dos primeros bienios de gobierno del Frente Amplio. De igual modo, los niveles de eficiencia (número de leyes aprobadas sobre proyectos enviados) también verifican una estabilidad sorprendente. Durante las dos presidencias, la tasa de aprobación del Ejecutivo sólo varía en un punto porcentual (de 54 a 55\%). Los cambios son leves y se registran fundamentalmente en la actividad de un Legislativo menos proactivo en la confección de proyectos de ley. En líneas generales, la comparación de los dos primeros bienios del Frente Amplio arroja el mismo nivel de eficiencia general en la aprobación de legislación (31\%). Esto es, cerca de uno de cada tres proyectos presentados se convierte en ley.

En el largo plazo, los dos gobiernos frenteamplistas están por encima de la media en la tasa de eficacia, cercana al 26\% (Gráfico 1). El punto más alto en la serie lo registra el primer bienio del gobierno del presidente Batlle, en el marco de una profunda crisis económica que forzó al Parlamento hacia una sintonía fina con el Poder Ejecutivo. De hecho, la crisis y la necesidad de salir rápidamente de ella con medidas de diverso tipo que implicaban nueva regulación, puso a prueba a todo el sistema político uruguayo y en particular al Parlamento. Esta hipótesis resulta plausible, dado que el presidente Batlle accedió al gobierno con una configuración de apoyo político extremadamente débil, dentro y fuera de su partido. Sin embargo, el Parlamento fue capaz de apoyarlo en una porción importante de la legislación propuesta por el Ejecutivo que buscaba salir de la crisis. Sin ella o lejos de la tormenta desatada en junio de 2002, el Frente Amplio ha logrado hacer funcionar su mayoría legislativa a los efectos de aprobar una porción importante de la agenda marcada por el presidente Mujica.

Con todo, también existen algunas diferencias destacables entre la capacidad de los Ejecutivos de aprobar legislación. Como muestran Chasquetti y Guedes (2011), los

Cuadro 6. Iniciativa Legislativa por Poder. Bienios 2005-2006; 2010-2011

\begin{tabular}{lccccccc}
\hline & \multicolumn{2}{c}{$\begin{array}{c}\text { Poder } \\
\text { Ejecutivo }\end{array}$} & \multicolumn{2}{c}{$\begin{array}{c}\text { Poder } \\
\text { Legislativo }\end{array}$} & \multicolumn{2}{c}{ Total } \\
\hline Proyectos presentados en 2005-2006 & 287 & $37 \%$ & 479 & $63 \%$ & 766 & $100 \%$ \\
Proyectos presentados en 2010-2011 & 290 & $43 \%$ & 383 & $57 \%$ & 673 & $103 \%$ \\
Leyes sancionadas en 2005-2006 & 158 & $66 \%$ & 80 & $34 \%$ & 238 & $100 \%$ \\
Leyes sancionadas en 2010-2011 & 158 & $76 \%$ & 51 & $24 \%$ & 209 & $100 \%$ \\
Eficacia* 2005-2006 & $55 \%$ & - & $17 \%$ & - & $31 \%$ & - \\
Eficacia 2010-2011 & $54 \%$ & - & $13 \%$ & $31 \%$ & - & 39 \\
\hline
\end{tabular}

* Número de proyectos aprobados sobre el total de proyectos presentados.

Fuente: Elaboración propia en base a datos del Sistema de Información Parlamentaria (SIP). 
Gráfico 1: Tasa de eficacia según gobierno: Uruguay 1985-2011

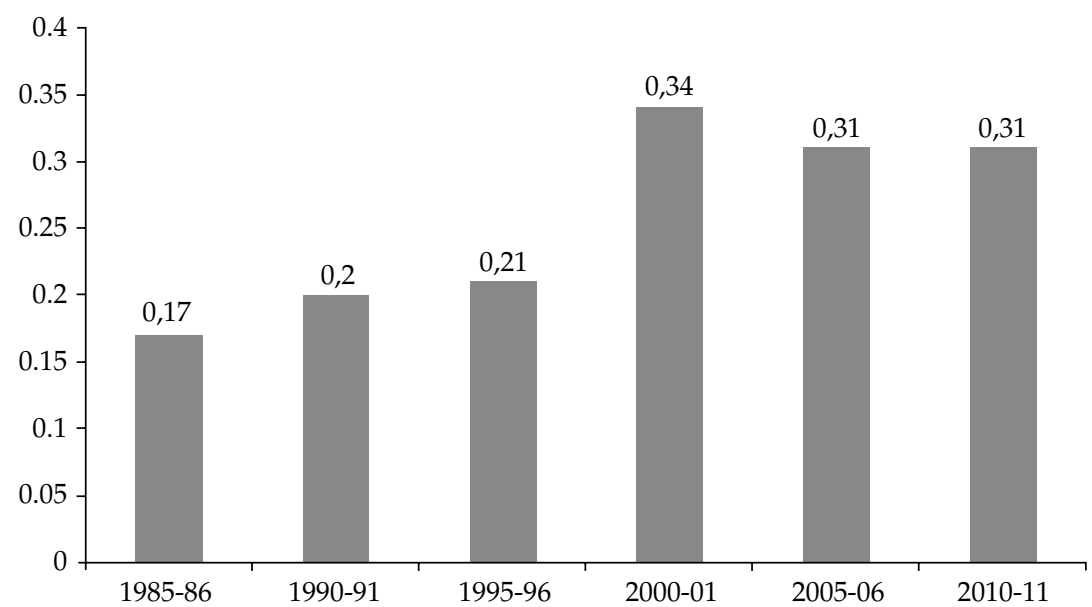

Fuente: Elaboración propia en base a datos de Chasquetti y Guedes 2011.

gobiernos del Frente Amplio con mayoría legislativa de partido son más exitosos en la aprobación de legislaci ón que la coalición mayoritaria de Colorados y Nacionalistas durante el gobierno del presidente Sanguinetti, y la mayor parte del gobierno en condición minoritaria durante la Presidencia del Dr. Jorge Batlle. Dicho esto, el éxito del Frente Amplio en el Legislativo no tiene costocero. Como muestran Chasquetti y Guedes (2011), los dos gobiernos del Frente Amplio con partido en mayoría muestran una interna partidaria con mayor propensión a modificar la legislación originada en el Poder Ejecutivo, comparado con el último gobierno Colorado del Presidente Batlle. En años recientes, la distancia ideológica dentro del Frente Amplio (entre las fracciones) ha sido mayor que la distancia entre los partidos Nacional y Colorado unidos en coalición.

\section{COMPITIENDO DESDE ABAJO: ELECCIONES SUBNACIONALES DE 2010}

El ciclo electoral uruguayo iniciado en octubre de 2009 con las elecciones legislativas y luego prolongado al ballotage por la elección presidencial de noviembre de ese mismo año, se vio extendido con la realización de las elecciones departamentales y municipales del 9 de mayo de 2010. ${ }^{9}$ Para las elecciones departamentales de 2010 a realizarse en los 19 departamentos del país, el Frente Amplio aspiraba a consolidar su crecimiento en el interior, mientras que los partidos Nacional y Colorado aspiraban a recuperar algunos departamentos que habían perdido en la anterior instancia electoral de 2004. Como puede apreciarse en el Cuadro 7, en todo el país el FA obtuvo el $42,4 \%$ de los votos, seguido del PN con el 30,9\%, el PC con el 15,0\%, el PI con el 0,8\%, y AP con el 0,7\%.

$9 \quad$ El nivel de gobierno municipal fue introducido por la Ley $\mathrm{N}^{\mathrm{o}} 18.567$, sancionada durante la administración Vázquez el 13 de setiembre de 2009, y parcialmente modificada por la Ley No 18.644 del 12 de febrero de 2010, y la No 18.653 del 15 de marzo del 2010, ambas durante la actual legislatura. 
Estos porcentajes son muy similares a los de la elección nacional de octubre de 2009, reflejando una pequeña pérdida de votos para el FA.

Las elecciones departamentales de 2010 significaron un terremoto dentro del Frente Amplio, luego de perder tres intendencias ganadas por primera vez en 2005. Lejos de acrecentar su apoyo electoral, el Frente Amplio vio disminuido su control político en el interior del país. Logró retener la intendencia de Montevideo, pero rodeada de un gran escepticismo y críticas de una porción importante de su electorado cautivo, que optó por votar en blanco en rechazo por la gestión del intendente Erlich y las características de la nominación de la actual intendenta Ana Olivera (Cardarello and Lorenzoni, 2011).

Por último, la creación del nivel de gobierno local (Municipios) constituye una novedad, tanto institucional como electoral para el período de referencia de este trabajo. A nivel institucional, la nueva legislación crea 89 municipios con sus respectivos alcaldes y concejales, con responsabilidades de gestión administrativa de unidades geográficas

Cuadro 7. Resultados electorales en elecciones departamentales de 2010 (porcentaje sobre el total de votos)

\begin{tabular}{|c|c|c|c|c|c|c|c|c|c|c|}
\hline Depto. & FA & PN & PC & PI & $\mathrm{AP}$ & $\begin{array}{c}\text { En } \\
\text { blanco } \\
\text { Total }\end{array}$ & $\begin{array}{c}\text { En } \\
\text { blanco } \\
\text { Parcial }\end{array}$ & $\begin{array}{l}\text { Sobres } \\
\text { c/ hojas } \\
\text { Anuladas }\end{array}$ & $\begin{array}{c}\text { Observados } \\
\text { y } \\
\text { Anulados }\end{array}$ & $\begin{array}{c}\text { Suma } \\
\text { votos } \\
\text { Emitidos }\end{array}$ \\
\hline Montevideo & 45,9 & 19,79 & 18,1 & 1,3 & 1,14 & 7,55 & 2,15 & 4,06 & 0,01 & 100 \\
\hline Canelones & 52,49 & 22,82 & 9,53 & 1,12 & 0,66 & 5,79 & 4,66 & 2,93 & 0,01 & 100 \\
\hline Maldonado & 47,97 & 30,52 & 12,66 & 0,9 & 0,16 & 2,94 & 3,04 & 1,8 & 0 & 100 \\
\hline Rocha & 52,48 & 30,11 & 7,76 & 0,44 & 0,53 & 3,91 & 3,08 & 1,68 & 0,02 & 100 \\
\hline T. y Tres & 40,76 & 49,71 & 5,33 & 0,29 & 0,25 & 1,75 & 0,83 & 1,07 & 0,01 & 100 \\
\hline Cerro Largo & 35,43 & 49,27 & 7,58 & 0 & 0,11 & 1,72 & 5,05 & 0,8 & 0,04 & 100 \\
\hline Rivera & 21,3 & 24,75 & 48,47 & 0,28 & 0 & 2,7 & 1,41 & 1,07 & 0,02 & 100 \\
\hline Artigas & 44,74 & 39,81 & 9,48 & 0,11 & 0,24 & 1,97 & 2,54 & 1,07 & 0,04 & 100 \\
\hline Salto & 40,33 & 12,45 & 41,93 & 0,71 & 0,13 & 2,47 & 0,94 & 1,02 & 0,02 & 100 \\
\hline Paysandú & 42,24 & 43,94 & 7,66 & 0 & 0,53 & 2,72 & 1,38 & 1,48 & 0,04 & 100 \\
\hline Río Negro & 36,77 & 42,37 & 15,5 & 0 & 0 & 2,47 & 1,51 & 1,36 & 0,03 & 100 \\
\hline Soriano & 31,89 & 53,87 & 6,08 & 0,54 & 0,3 & 3,02 & 2,91 & 1,36 & 0,04 & 100 \\
\hline Colonia & 30,56 & 51,19 & 10,05 & 0 & 0,15 & 2,77 & 3,58 & 1,68 & 0,02 & 100 \\
\hline San José & 32,42 & 55,87 & 3,9 & 0,64 & 0,47 & 3,29 & 1,88 & 1,51 & 0,02 & 100 \\
\hline Flores & 23,51 & 59,58 & 10,24 & 0,67 & 0 & 3,63 & 0,84 & 1,53 & 0 & 100 \\
\hline Florida & 41,74 & 42,24 & 10,28 & 0 & 0,29 & 2,8 & 1,19 & 1,43 & 0,03 & 100 \\
\hline Durazno & 26,38 & 60,99 & 6,42 & 0,75 & 0,38 & 2,78 & 1,02 & 1,25 & 0,05 & 100 \\
\hline Lavalleja & 32,04 & 52,94 & 8,58 & 0,64 & 0,11 & 3,1 & 1,27 & 1,32 & 0 & 100 \\
\hline Tacuarembó & 23,23 & 67,26 & 5,13 & 0 & 0 & 2,19 & 1,35 & 0,81 & 0,03 & 100 \\
\hline Total & 42,36 & 30,9 & 15,01 & 0,84 & 0,65 & 5,08 & 2,5 & 2,64 & 0,02 & 100 \\
\hline
\end{tabular}

Fuente: Elaboración propia en base a datos de la Corte Electoral. 
con distinto tamaño y población. A nivel electoral, de los 89 municipios en disputa, 41 fueron obtenidos por el Frente Amplio, 42 por el Partido Nacional y 6 por el Partido Colorado. Nótese que las elecciones para este tercer nivel de gobierno se realizaban en forma concomitante con las elecciones departamentales y de hecho compiten los mismos partidos que en la escena departamental y nacional. Sin embargo, estas primeras elecciones a nivel municipal estuvieron teñidas por un gran nivel de incertidumbre entre los votantes, derivado de la falta de información y problemas de coordinación y movilización de los partidos. Ello explica, al menos en parte, el sorprendente porcentaje de votos en blanco, cercano al $40 \%$ del electorado (para aquellos que decidieron votar en el nivel departamental sin el nivel municipal), además de aquellos que decidieron votar en blanco en el nivel municipal y departamental (6,3\%).

\section{CONSIDERACIONES FINALES}

A dos años de iniciado el mandato del presidente Mujica, 2012 constituye una bisagra para el actual gobierno del Frente Amplio. El propio Presidente ha visto el inicio de 2012 como un desafío importante para su gestión, dado que varios asuntos de la agenda se han visto seriamente enlentecidos por diversas circunstancias. Entre 2010 y 2011, el gobierno ha verificado algunas dificultades en la gestión de la agenda y en particular de los tópicos considerados prioritarios por el Presidente. Por su parte, la oposición también ha enfrentado dificultades importantes no sólo de diferenciación entre los partidos Nacional y Colorado, sino también de cooperación para competir contra el gobierno. Desde el inicio del gobierno del presidente Vázquez, el Frente Amplio ha adoptado en forma estable un conjunto de políticas económicas que lo acercan a las preferencias de la oposición. Esto ha dificultado notoriamente la competencia de la oposición con el gobierno. Adicionalmente, el gobierno del presidente Mujica ha buscado una serie de acuerdos en áreas importantes de política pública como la educación, la energía, la seguridad y más recientemente la salud pública. El resultado de estos acuerdos ha sido muy limitado, pero en esencia reducen aún más las posibilidades de competencia, para una oposición que ya ha visto reducido su campo de acción con el curso que el Frente Amplio le ha dado a la política económica. Con un escenario económico internacional incierto (si no preocupante), escasos logros en materia de acuerdos con la oposición y dificultades en el manejo de la agenda de gobierno (dentro de su partido), el presidente Mujica encara la segunda mitad de su mandato con una preocupación justificada.

\section{REFERENCIAS}

Amorim Neto, Octavio. 1998. Cabinet formation in Presidential Regimes: An Analysis of 10 Latin American Countries. XX Congreso de LASA. Chicago: 26 p.

Bentancur, Nicolás y María E. Mancebo. 2011. "La educación en la encrucijada: Escrutinio Político y Gobierno de la Enseñanza". "Política en Tiempos de Mujica: En Busca del Rumbo". En Informe de Coyuntura $N^{\circ} 10,124-130$. Instituto de Ciencia Política. Estuario Editora. 
Buquet, Daniel y Diego Luján. 2011. “El Poder Ejecutivo". En "Política en Tiempos de Mujica: En Busca del Rumbo". Informe de Coyuntura $\mathrm{N}^{\circ} 10,183-193$. Instituto de Ciencia Política. Estuario Editora.

Buquet, Daniel. 2009. Uruguay 2008: de las reformas a la competencia electoral. Santiago de Chile, Revista de Ciencia Política 29 611-632.

Cardarello, Antonio y Miguel Lorenzoni. 2011. Estrenando la doble escena subnacional: Elecciones departamentales y municipales. En "Política en Tiempos de Mujica: En Busca del Rumbo". Informe de Coyuntura $N^{\circ} 10,257-267$. Estuario Editora.

Chasquetti, Daniel. 2007. Uruguay 2006: éxitos y dilemas del gobierno de izquierda. Santiago de Chile, Revista de Ciencia Política: 249-263.

Chasquetti, Daniel. 2008. Uruguay 2007: el complejo año de las reformas, Revista de Ciencia Política. 28: 385-403.

Chasquetti, Daniel y Alejandro Guedes. 2011. El primer año legislativo del gobierno de Mujica. En "Política en Tiempos de Mujica: En Busca del Rumbo". Informe de Coyuntura Nº 10,194-209. Estuario Editora.

Garcé, Adolfo. 2010. Uruguay 2009: de Tabaré Vázquez a José Mujica, Revista de Ciencia Política 30: 499-535.

Kernell, Samuel. 1993. Going Public: New Strategies of Presidential Leadership. Washington D.C., Brookings Institution.

Magar, Eric y Juan Andrés Moraes. 2012. "Factions with clout: Presidential cabinet coalition and policy in the Uruguayan parliament". Party Politics forthcoming: 25.

Selios, Lucía. 2011. Opinión Pública. En "Política en Tiempos de Mujica: En Busca del Rumbo". Informe de Coyuntura $N^{\circ} 10,211-230$. Estuario Editora.

Vila, Alejandro. 2011. Seguridad Ciudadana: ¿continuidad o cambio? En "Política en Tiempos de Mujica: En Busca del Rumbo". Informe de Coyuntura $N^{\circ} 10,159-166$. Estuario Editora.

Juan Andrés Moraes. Profesor adjunto en el Departamento de Ciencia Política de la Universidad de la República. Es coautor con Daniel Buquet y Daniel Chasquetti del "Enfermo Imaginario" (FCS, 1998) así como otras publicaciones en Penn State University Press (2008), Harvard University Press (2009), Comparative Political Studies (2009) y Party Politics (en prensa).

Email: jmoraes@fcs.edu.uy

Alejandro Guedes. Asistente de investigación en el Departamento de Ciencia Política de la Universidad de la República.

Email: neryguedes@gmail.com

Diego Luján. Asistente de investigación en el Departamento de Ciencia Política de la Universidad de la República.

Email: dlujan@fcs.edu.uy 
